\title{
Multijet events and parton showers
}

\author{
F. Krauss ${ }^{1,2 *}$, R. Kuhn ${ }^{1,2 \dagger}$, G. Soff ${ }^{1}$ \\ ${ }^{1}$ Institut für Theoretische Physik, TU Dresden, 01062 Dresden, Germany, \\ ${ }^{2}$ Max-Planck-Institut für Physik komplexer Systeme, 01187 Dresden, Germany
}

\begin{abstract}
We propose a general approach for the description of multijet events in the framework of QCD event generators. We introduce a new algorithm to match parton showers and arbitrary matrix elements for the production of any number of jets via the strong or electroweak interaction. We developped a new parton cascade code capable to deal with multijet events at LEP energies and beyond.
\end{abstract}

PACS numbers 13.65.+i

The formation of jets in $e^{+} e^{-}$annihilations provides an interesting testing ground for many areas in modern particle physics. In the past years especially the production of four jets has drawn considerable attention for several reasons.

First, the interpretation of these events by means of the processes

$$
e^{+} e^{-} \rightarrow q \bar{q} q^{\prime} \bar{q}^{\prime}, \quad e^{+} e^{-} \rightarrow q \bar{q} g g
$$

respectively, allows for a test of the symmetry group underlying the strong interaction [1] via their colour factors $C_{A}, C_{F}$ and $T_{F}$ [2]. Second, since the corresponding cross sections depend sensitively on the number of flavours active at the relevant scale, effects of new physics might be constrained by their precice determination [3]. Third, four jet events represent a serious background to the production and decay of pairs of electroweak gauge bosons and of the Higgs boson of the Standard Model [4]. Last but not least, these processes might provide additional signatures for new particles.

This phenomenological impact clearly signals the need for an adequate and thorough description of four and more jet events and a proper implementation into a multiple purpose Monte Carlo event generator.

In principle, multijet topologies like the ones exemplified above, Eq. (11), are well described by the corresponding matrix elements evaluated in the standard way of summing and squaring amplitudes. Unfortunately the final states accessible by this method are not the hadrons observed experimentally but partons. Therefore some hadronization scheme has

*E-mail: krauss@theory.phy.tu-dresden.de

†E-mail: kuhn@theory.phy.tu-dresden.de 
to be used with usually scale dependent parameters. Thus perturbative jet evolution as modelled by the parton shower approach is an important feature of event generators since it allows for the construction of a process independent infrared scale of the order of a few $\Lambda_{\mathrm{QCD}}$ defining the onset of hadronization and consequently guaranteeing its universality. This explains the need for a general procedure to match all possible matrix elements for the various channels of jet production and the parton shower [5] [7].

Before discussing in some detail the matching procedure used within the new event generator $\mathrm{APACIC}++$ we would like to summarize the relevant characteristics of matrix elements and parton showers.

As indicated above the matrix elements are related to specific processes, in our case to processes like the one of Eq. (1), describing the hard production of a number of partons. They can be evaluated perturbatively in the standard way, results for the production of four jets via the strong interaction exist in Leading [8] and Next-to Leading Order [9]. Here we do not want to comment on the pecularities related to the mutual cancellation of virtual and real divergencies occuring in the perturbative treatment of the matrix elements. However, we would like to stress, that the real divergencies related to soft and collinear emissions can be avoided by the requirement to describe exclusive processes, i.e. the production of a number of jets instead of partons. Popular schemes used for the definition of jets are the JADE- [10] and DURHAM-algorithm [11. At this point it should be noted that the matrix elements describe on-shell particles in the final state lacking the possibility to radiate further and produce a jet.

The jet evolution is commonly modelled by the parton shower approach. Within the presentation of the matching algorithm employed by our code APACIC++ we refer to a parton shower ordered by virtualities with an angular veto on growing branching angles [12] to account for the proper internal jet structure. Additionally, we take all partons massless. However, an extension to a parton shower ordered by angles [13] and massive partons is straightforward. Within this framework, the virtualities of the radiating partons play the role of an order parameter organizing the multiple emissions of the shower approach via the corresponding Sudakov form factor [14]

$$
\Delta(t)=\exp \left[-\int_{t_{0}}^{t} \frac{\mathrm{d} t^{\prime}}{t^{\prime}} \int \mathrm{d} z \frac{\alpha_{s}\left(\mu\left(t^{\prime}, z\right)\right)}{2 \pi} \hat{P}(z)\right] .
$$

Note, that the splitting function $\hat{P}(z)$ entering the Sudakov form factor is derived for massless on-shell particles in the final-state. To account for local four-momentum conservation one commonly changes the four momenta of the outgoing particles to correct for effects related to the virtual masses they pick up in their subsequent decays, for details see [15]. In this respect, the virtualities represent a minor perturbation of the branching kinematics.

The construction of a parton shower relies on an expansion around the soft and collinear limits of the emission and the angular ordering accounts for the coherence of the shower. Taken together, both features allow for a construction of jets by means of subsequent independent parton emissions. Even the inclusion of azimuthal correlations steming from the helicity structure involved in two subsequent emissions does not alter this picture drastically [16]. Therefore it should be obvious that the parton shower approach is not capable of modelling the complicated topological structure of multijet events and one has to rely on the matrix elements and match the parton shower. 
According to the considerations above the problem to match the matrix elements to the parton shower can be reformulated as the question of how to supply the final-state partons of the matrix elements with virtual masses in a sensible way.

We now turn to the discussion of our matching procedure once a kinematical configuration of initial partons, i.e. jets, is given. Our approach basically requires the production of a jet to be a hard process modelled by the corresponding matrix elements and the jet evolution to be governed by the parton shower. Following the reasoning of [6] we therefore separate the scales for jet production and the onset of jet evolution appropriately. In difference to their procedure to define the separation scale by a suitable choice of a fixed $Q^{2}$ as the initial virtual mass of every parton giving rise to a jet, however, we prefer to use the corresponding Sudakov form factor to determine this virtuality, i.e. the invariant mass of the resulting jet. The choice of the Sudakov form factor to govern the virtual masses of the initial partons results in varying invariant masses steming from the hard process and a smoother transition of topologies involving different numbers of jets. Of course the number of jets in a specific event can be chosen according to the perturbative rate.

Since the Sudakov form factor enters the parton shower in the form

$$
\mathcal{P}_{\text {no }}=\frac{\Delta\left(t_{i j}\right)}{\Delta\left(t_{i}\right)}
$$

determining the probability of no resolvable parton emission between $t_{i j}$ and $t_{i}$ we have to construct the virtual masses $t_{i j}=\left(p_{i}+p_{j}\right)^{2}$ of the intermediate states $i j$ the outgoing partons $i$ and $j$ originate from. Equipped with this intermediate virtual masses we are able to construct the virtualities $t_{i}$ and $t_{j}$ of the initial partons $i$ and $j$ via the corresponding Sudakov form factor of Eq. (3). The task left is now to determine these intermediate virtualities. In most cases, a number of Feynman diagrams contributes to the matrix element under consideration. Thus one has to define relative probabilities of the various parton histories leading to different $t_{i j}$ allowing to chose one of the histories by random.

The algorithm described above can be best illustrated by considering the production of four jets as indicated in Eq. (1). Seven Feynman diagrams contribute and in a first step one choses the flavours of the four partons by random according to the corresponding cross sections. This is in full analogy to the determination of the number of jets.

Let us assume in the following, that we have chosen the $q \bar{q} g g$-configuration and that we have constructed the momenta of the outgoing partons according to the corresponding differential distribution. So we are now able to construct relative probabilities of the five histories or diagrams $i$ using the appropriate amplitudes $\mathcal{M}_{i}$ via

$$
\mathcal{P}_{i}=\left|\mathcal{M}_{i}\right|^{2} .
$$

Alternatively one might reconstruct the individual parton histories in a fashion motivated by the parton shower [5]. For example, in this approach the probability of the history depicted in Fig. 囬 reads

$$
\mathcal{P}=\mathcal{P}_{1 \rightarrow 34} \mathcal{P}_{4 \rightarrow 56}=\frac{1}{t_{1}} P_{q g}\left(z_{34}\right) \frac{1}{t_{4}} P_{g g}\left(z_{56}\right),
$$

where the $P(z)$ are the well-known splitting functions related to the branching processes involved, the $t_{i}$ are the squares of the four momenta as given by 


$$
\begin{aligned}
& t_{1}=p_{1}^{2}=\left(p_{3}+p_{5}+p_{6}\right)^{2} \\
& t_{4}=p_{4}^{2}=\left(p_{5}+p_{6}\right)^{2}
\end{aligned}
$$

and the $z_{i}$ are defined by

$$
\begin{aligned}
z_{b c} & =z_{a \rightarrow b c}=\frac{t_{a}}{\lambda} \frac{E_{b}}{E_{c}}-\frac{t_{a}-\lambda+t_{b}+t_{c}}{2 \lambda}, \\
\lambda & =\sqrt{\left(t_{a}-t_{b}-t_{c}\right)^{2}-4 t_{b} t_{c}} .
\end{aligned}
$$

Having chosen one of the histories according to the relative probabilities it is straightforward to construct the virtual masses of the intermediate states previously denoted as $t_{i j}$. In our example, Fig. 1, this amounts to calculate $t_{4}=p_{4}^{2}\left(p_{5}+p_{6}\right)^{2}$ and $t_{1}=p_{1}^{2}=\left(p_{3}+p_{4}\right)^{2}$.

Now the Sudakov form factor determines the virtual mass of the outgoing particle under the requirement that its decay does not produce any additional jet. The kinematical distribution of the final state particles of the matrix element is corrected in the manner employed within the parton shower to conserve the total four momentum at this place, too.

Two further remarks are in order here. First, it should be noted that our matching procedure of parton showers and matrix elements relies on an internal jet-clustering algorithm and yields sensible results for observables related to jets as long as we produce them only perturbatively. Other observables do not depend sensitively on this internal clustering scheme. Second, to account for the appropriate jet-rates it should be mentioned that so far the scale of the couplings and especially the scale of $\alpha_{s}$ within the matrix element expressions has not been fixed. We propose to use an effective scale $s_{\text {eff }}=s \kappa_{s}$ for the various coupling constants. With a suitable choice of the scalefactor $\kappa_{s}$ rates for different numbers of jets at various energies can be accounted for simultaneously. This enables us to treat the QCD-production of varying numbers of jets on equal footing.

We have performed a comparison of a variety of observables describing the event shapes of three- and four jet-events at energies between 91 and $161 \mathrm{GeV}$ at the level of matrix elements, parton showers and hadrons using PYTHIA [15], HERWIG [17] and our event generator $\mathrm{APACIC}++$. No initial state radiation was taken into account. We have used the matrix element expressions for the production of up to four jets as provided by the code of [18 and the hadronization scheme of [19] as provided by JETSET linked to our parton shower.

The results for most of the observables obtained with the different event generators coincide nicely with each other and with experimental data 20 22]. For a representative extract of various event shape observables see Fig. 2. At this place it should be noted that we used the tuned parameters for the other event generators as given in 23] representing the best choice to account for the experimental data. In contrast the parameters of our code have not been fitted so far and we used the unaltered hadronization parameters of PYTHIA. This somewhat lowers the quality of the results produced by APACIC++. Nevertheless, the results of $\mathrm{APACIC}++$ are in fair agreement with data indicating that our approach to match matrix elements and parton showers is perfectly capable to describe the interplay of various numbers of jets as well as the overall features of $e^{+} e^{-}$events.

However, the validity of our ansatz can be verified when considering the topological structures of multijet events as exemplified by four-jet events. Ordering the jets by their energies, $E_{1} \geq E_{2} \geq E_{3} \geq E_{4}$, typical observables describing these processes are the modified 
Nachtmann-Reiter-, the Körner-Schierholz-Willrodt- and the Bengtson-Zerwas-angle as well as $\alpha_{34}$ [1, 24],

$$
\begin{aligned}
\theta_{\mathrm{NR}}^{*} & =\angle\left(\overrightarrow{p_{1}}-\overrightarrow{p_{2}}, \overrightarrow{p_{3}}-\overrightarrow{p_{4}}\right), \\
\chi_{\mathrm{BZ}} & =\angle\left(\overrightarrow{p_{1}} \times \overrightarrow{p_{2}}, \overrightarrow{p_{3}} \times \overrightarrow{p_{4}}\right), \\
\theta_{34} & =\angle\left(\overrightarrow{p_{3}}, \overrightarrow{p_{4}}\right), \\
\Phi_{\mathrm{KSW}}^{*} & =\angle\left(\overrightarrow{p_{1}} \times \overrightarrow{p_{3}}, \overrightarrow{p_{2}} \times \overrightarrow{p_{4}}\right),
\end{aligned}
$$

where the last defintion holds for $\left|\overrightarrow{p_{1}}+\overrightarrow{p_{3}}\right| \geq\left|\overrightarrow{p_{1}}+\overrightarrow{p_{4}}\right|$ and in the opposite case we interchange $\vec{p}_{3}$ and $\vec{p}_{4}$.

In Fig. 3 we show the angular distributions of the partons after the shower generated by the various event generators in comparison to the distributions as given by the matrix element. Obviously APACIC ++ is perfectly capable to describe the four jet topologies in detail since the parton shower and the matrix element are matched appropriately. The only sizeable deviations of the jet distributions after the parton shower from the result as given by the matrix element alone are centered in the region of nearly collinear jets. This is not too surprising, however, since the jet evolution widens the initial partons to jets and therefore overshadows the jet definition of two jets very close to each other.

In contrast, the two other event generators do not include an accurate matching procedure for events with more than three jets. Therefore, their parton shower results fail to describe any four- or more jet specific topological structures. So the large deviations for nearly the whole angular region under consideration merely reflect the fact that the parton shower alone is not capable to model the rich topological structure of multijet events.

We have proposed a general approach to match parton showers and arbitrary matrix elements in the framework of QCD event generators. The results obtained with the help of our algorithm show a fair agreement with experimental data and matrix element expressions available and are almost insensitive to internal parameters within reasonable regions. An extension to jets produced via the electroweak interaction is straightforward.

In this respect, our approach offers new possibilities to describe some precision data concerning multijet events at LEP II and beyond.

\section{ACKNOWLEDGEMENTS}

We would like to thank B. Ivanyi for lively and helpful discussions. We gratefully acknowledge financial support by DFG, BMBF and GSI. 


\section{REFERENCES}

[1] J. G. Körner, G. Schierholz, J. Willrodt, Nucl. Phys. B185 (1981) 365.

[2] See for example:

R. D. Field, Applications of perturbative QCD, Addison-Wesley Publishing, Reading (Mass.) (1989).

[3] Consider for example the production of gluinos:

G. L. Kane, W. B. Rolnick, Nucl. Phys. B217 (1983) 117.

[4] W. Beenakker et al., Physics at LEP2, vol. 1 79-139, hep-ph/9602351.

[5] J. Andre, T. Sjostrand, Phys. Rev. D57 (1998) 5767; J. Andre, hep-ph/9706325

[6] M. H. Seymour, Comp. Phys. Commun. 90 (1995) 95.

[7] S. Moretti, W. J. Stirling, hep-ph/9808429.

[8] R. K. Ellis, D. A. Ross, A. E. Terrano, Nucl. Phys. B178 (1981) 421; W. T. Giele, E. W. N. Glover, Phys. Rev. D46 (1992) 1980.

[9] See for example:

Z. Bern, L. Dixon, D. A. Kosower, S. Weinzierl, Nucl. Phys. B489 (1997) 3; Z. Bern, L. Dixon, D. A. Kosower, Nucl. Phys. B513 (1998) 3; A. Signer, L. Dixon, Phys. Rev. Lett. 78 (1997) 811; Z. Nagy, Z. Trocsanyi, Phys. Rev. D57 (1998) 5793.

[10] Jade-Collaboration, Z. Phys. C33 (1986) 235; Jade-Collaboration, Phys. Lett. B213 (1988) 235.

[11] S. Catani, Y. L. Dokshitser, M. Olsson, G. Turnock, B. R. Webber, Phys. Lett. B269 (1991) 432; Y. L. Dokshitser, J. Phys. G17 (1991) 1537.

[12] M. Bengtsson, T. Sjöstrand, Phys. Lett. 185B (1987) 435; M. Bengtsson, T. Sjöstrand, Nucl. Phys. B289 (1987) 810.

[13] Y. L. Dokshitser, S. I. Troyan, Proceedings of the XVII Winter School of the LNPI (1982); Yu. L. Dokshitser, V. A. Khoze, S. I. Troyan, Coherence and physics of QCD jets in Perturbative Quantum Chromodynamics, ed.: A. H. Mueller, World Scientific, Singapore (1989).

[14] V. V. Sudakov, Sov. Phys. JETP 30 (1956) 65.

[15] T. Sjöstrand, Comp. Phys. Commun. 39 (1984) 347; M. Bengtsson, T. Sjöstrand, Comp. Phys. Commun. 43 (1987) 367; T. Sjöstrand, Comp. Phys. Commun. 82 (1994) 74.

[16] B. R. Webber, Ann. Rev. Nucl. Part. Sci 36 (1983) 201.

[17] G. Marchesini, B. R. Webber, G. Abbiendi, I. G. Knowles, M. H. Seymour, L. Stanco, Comp. Phys. Comm. 67 (1992) 465; G. Marchesini, B. R. Webber, G. Abbiendi, I. G. Knowles, M. H. Seymour, L. Stanco, hep-ph/9607393.

[18] Z. Nagy, Z. Trocsanyi, Phys. Rev. Lett. 79 (1997) 3604;

[19] B. Andersson, G. Gustafson, G. Ingelmann, T. Sjöstrand, Phys. Rep. 97 (1983) 33.

[20] The ALEPH-Collaboration, Phys. Rep. 294 (1998) 1.

[21] The DELPHI-Collaboration, Z. Phys. C56 (1992) 63.

[22] The L3-Collaboration, Z. Phys. C55 (1992) 39; Phys. Lett. B404 (1997) 390

[23] K. Hamacher, M. Weierstall; hep-ex/9511011

[24] O. Nachtmann, A. Reiter, Z. Phys. C16 (1982) 45; M. Bengtsson, P. M. Zerwas, Phys. Lett. B208 (1988) 306; S. Moretti, J. B. Tausk, Z. Phys. C69 (1996) 635. 


\section{FIGURES}

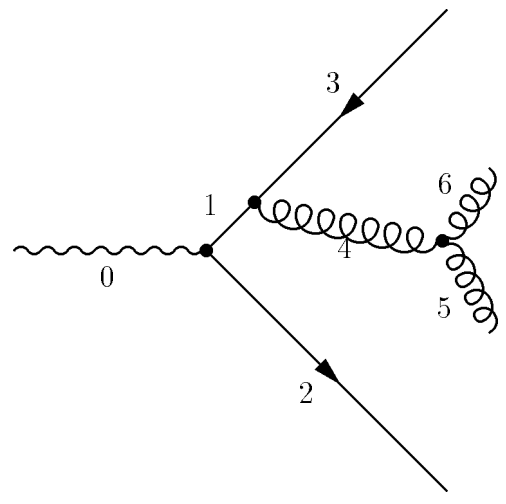

FIG. 1. Typical graph for $e^{+} e^{-} \rightarrow$ four jets at leading order.
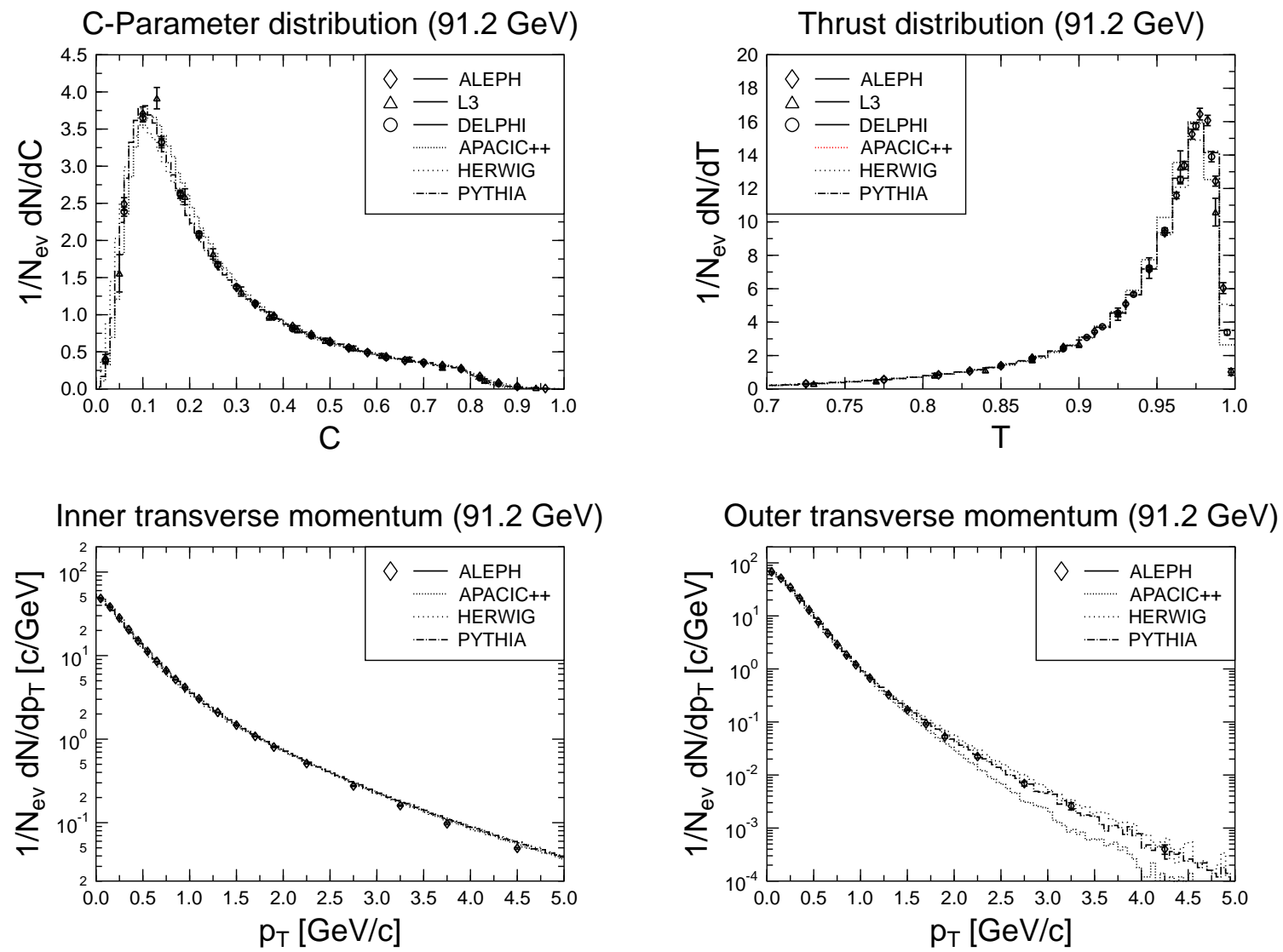

FIG. 2. Comparison of experimental data and event generators for a variety of event shape observables at the hadron level at the $Z$-pole. For the hadronization the default parameters of PYTHIA [15] and HERWIG [17] were used. APACIC++ employed the Lund-String hadronization of PYTHIA. 

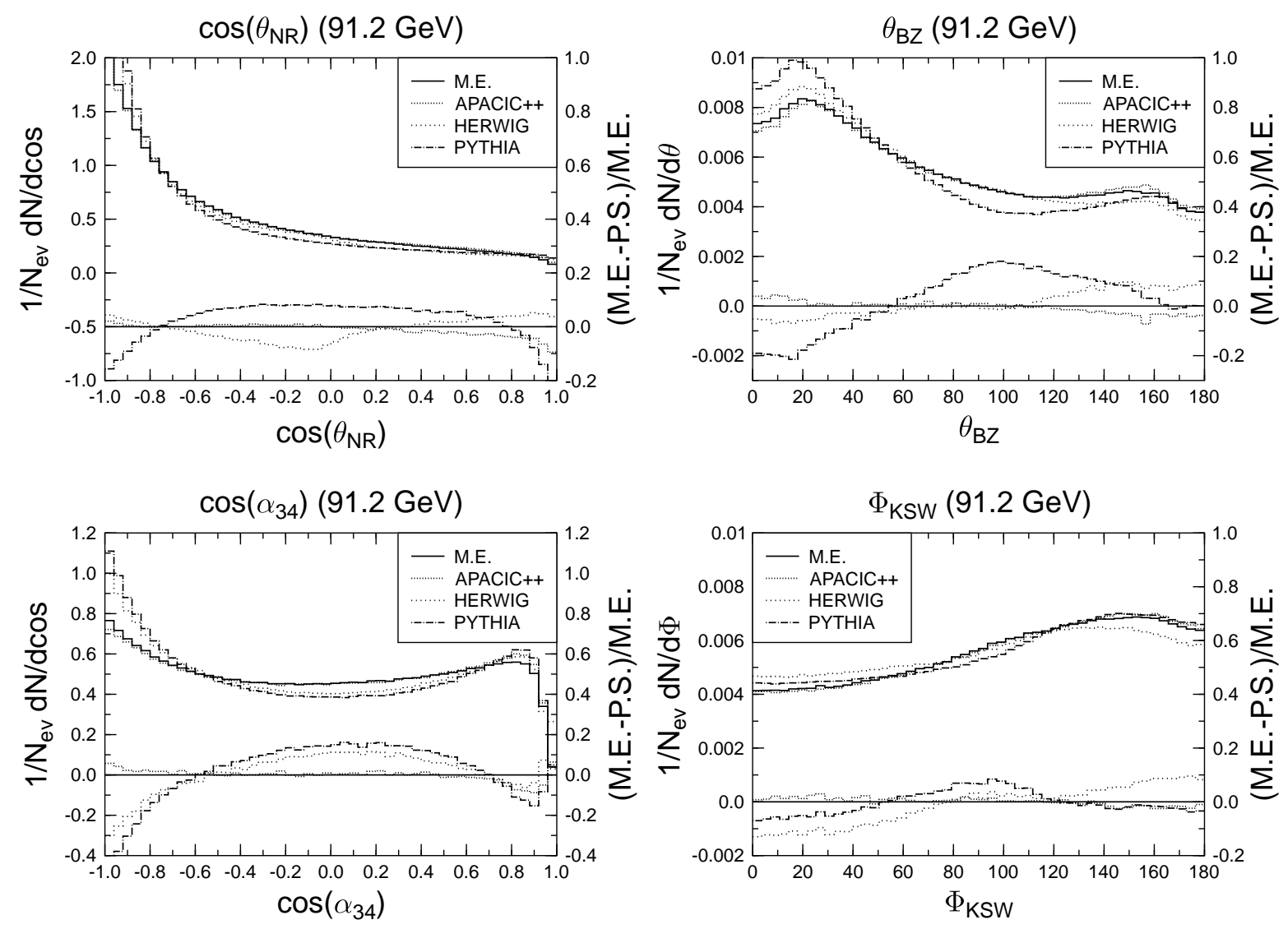

FIG. 3. Distributions for the various angles given in Eq. (8) as described by the matrix elements supplied by DEBRECEN [18] and the different event generators at the $Z$-pole. For the definition of jets the Durham-scheme with $y_{\text {cut }}=0.002$ was employed for all final states as well as for the matching of the matrix elements and the parton shower. The upper lines show the corresponding differential rates with respect to the numbers on the left axis whereas the errors relative to the matrix element expression are are given by the appropriate lower lines with respect to the numbers on the right axis. 